codon within the vertebrate lineage, and in one case a descendant of the intermediate non-serine protease (haptoglobin) can be found (see figure). This model also predicts that additional serine proteases may have lost protease function at some point in their evolutionary history, but due to back-mutations (restoring the serine codon to the ancestral type) there is no trace of their non-protease past in their present sequence, in contrast to the AGY serine proteases. The existence of tandem arrays within the family of more than 100 serine protease genes in the vertebrates has probably provided many opportunities for these genes to lose and regain protease function as well as to adapt to new functions.

Department of Biochemistry,

David M. IRWIN

University of California, Berkeley,

California 94720, USA

1. Brenner, S. Nature 334. 528-530 (1988)

2. Irwin, D.M. Robertson, K.A. \& MacGillivray, R.T.A.J. molec. Biol. 200, 31-45 (1988).

Doolittle, R.F. \& Feng. D.F. Cold Spring Harb. Symp. quant Biol 52 869-874 (1987)

4 Sadler J.E. Malinowski. D.P. \& Davie. E.W. in Progress in Fibrinolysis Vol. 7 (eds Davidson, J.F.. Donati, M. B. \& Coccheri, S.) 201-204 (Churchill Livingstone. Edinburgh. 1985).

5. Højrup, P., Jensen, M.S. \& Petersen, T.E. FEBS Lett. 184. 333-338(1985).

6. McLean, J.W. et al. Nature 330. 132-137 (1987).

\section{Universal and essential role of $\mathrm{MPF} / c d c 2^{+}$}

SIR-As reported recently in News and Views', the product of the mitosisinducing $c d c 2^{+}$gene of the fission yeast, Schizosaccharomyces pombe is a component of the maturation promoting factor $(\mathrm{MPF})^{2-4}$, first recognized in Xenopus oocytes. This exciting finding demonstrates the universal and essential roles of $\mathrm{MPF} / c d c 2^{+}$in cell-cycle regulation.

We previously isolated a meiosisspecific recessive mutant $t w s 1-N 22$ of $S$. pombe which seems to be normal in meiosis I but unable to enter into meiosis II so that resulting asci contain two viable diploid spores instead of the normal four haploid spores ${ }^{5,6}$. Mitotic growth of $t w s l$ is normal at any temperature. More recently we have found that tws 1 is an allele of the $c d c 2$ gene. Tetrad analysis shows that the tws 1 locus is tightly linked to $c d c 2$ (less than $1 \mathrm{cM}$ separates them). Cloned $c d c 2^{+}$ can complement $t w s l$, resulting in the formation of four-spored asci. Furthermore, mitotic growth of the $c d c 2-33^{\prime \prime} / t w s 1$ heterozygote is poor at non-permissive temperature, suggesting an interaction between their products or a shortage of the active $c d c 2$ product in the heterozygote. Temperature-sensitive alleles of $c d c 2$ also frequently produce two-spored asci at semi-permissive temperature ${ }^{7}$. The $t w s 1$ allele in $c d c 2$ might be meiosis-specific, such that the mutation specifically impairs a transition step to meiosis II. Alternatively, it might result in a reduced $c d c 2$ activity that is still sufficient for mitosis and meiosis I, but not meiosis II. In any case, the phenotype of tws $I$ allele has revealed another aspect of the various roles of the $c d c 2^{+}$gene in mitosis and meiosis.

Osami Niwa

Mitsuhiro Yanagida

Department of Biophysics,

Faculty of Science, Kyoto University,

Sakyo-ku, Kyoto 606, Japan

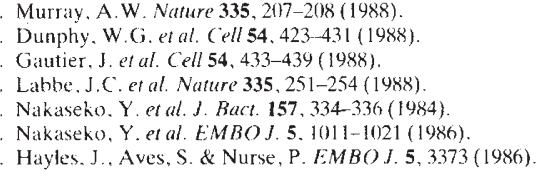

Murray, A.W. Nature 335, 207-208 (1988). Dunphy, W.G. et al. Cell 54, 423-431 (1988).

Gautier. J et al. Cell 54, 433-439(1988).

Labbe. J.C. et al. Nature 335, 251-254 (1988)

Labbe. J.C. et al. Nature 335, 251-254 (1988).
Nakaseho. Y et al. J. Bact. 157, 334-336 (1984).

Nakaseho. Y. et al. J. Bact. 157, 334-336 (1984).

Nakaseko, Y. el al. EMBOJ. P. EMBO J. 5,3373 (1986)

Hayles. J., Aves, S. \& Nurse, P. KMBO J. 5, 3373 (1986).

\section{Licence to slang Copenhagen revoked}

SIR-Posiewnik and Pykacz ${ }^{1.2}$ have described an interesting gedankenexperiment for which they claimed that the predicted result would depend on whether one employs the Copenhagen or the stochastic (the Einstein-De Broglie) interpretation of quantum mechanics. But even though the experiment seems to be perfectly realizable with optical devices now available (Physics Letters), one should not expect a scientific revolution soon.

The idea of the Posiewnik-Pykacz experiment boils down to the following. One performs a single photon Young's double-slit experiment with only one slit open at a time, and tries to get interference. More specifically, one has single photons in a Mach-Zehnder-type interferometer ${ }^{3}$. Each of the two possible paths of the photon can be blocked by a shutter. The shutters operate so that only one of them can be open at a time (which can be achieved in a relativistically invariant way). Interference can occur only when the parts of the photon wavepacket chopped by the shutters overlap in the detection region. This can be achieved by a suitable lengthening of the interferometer path with the shutter which opens first, which has the effect of delaying the part of the wave representing the photon traversing that path.

This outline description conforms to our intuition, as well as to the EinsteinDe Broglie interpretation of quantum mechanics. But despite the claim of Posiewnik and Pykacz, the introduction of the collapse postulate does not change the predictions. The Copenhagen point of view should read as follows. The collapse of a wavepacket represents a gain of information about a quantum object. But the experiment has been devised in such a way that if the photon passes through the shutters, we know only that it has passed through one of them. This is because the two paths are of different optical length. Thus any time correlation between the gate at a shutter and photon's arrival at a detector (placed at the end of both paths) is wiped out by the time delay. Indeed, interference is a maximum when the time delay is such that from the point of view of the detector, both shutters are 'seen' by it to open and close together.

Posiewnik and Pykacz seem to rely on the simple argument that, because there is a collapse, there can be no interference. But, one must know into what the wavepacket collapses. While, in those versions of Young's experiments presented in Feynman's lectures, the collapse indeed precludes interference, that is not so for the proposition of Posiewnik and Pykacz. MAREK ZuKOWSKI Uniwersytet Gdanski, Instytut Fizyki Teoretycznej i Astrofizyki, 80-952, Gdansk, Poland

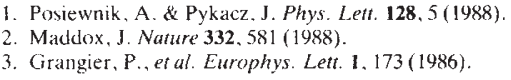

\section{Cystic fibrosis advantage}

SIR-In 1982, Quinton' presented evidence that anion impermeability is a basic defect in cystic fibrosis (CF), and speculated that "intestinal secretions of persons carrying the mutant CF gene may be less than those of the normal population when stimulated by cholera toxin... Consequently, these persons... should be at some advantage...". This proposal was recently repeated ${ }^{2}$, and several recent findings providing support for Quinton's speculation have also been cited or presented ${ }^{3}$. But a key finding germane to the argument was not cited. Because $\mathrm{CF}$ homozygotes reproduce only rarely, a selective advantage for the $\mathrm{CF}$ allele requires that it results in an altered phenotype when present as a single copy in heterozygotes. The laboratories have now demonstrated proportionally reduced fluid secretion by $\mathrm{CF}$ heterozygotes for $\beta$-adrenergically stimulated sweating ${ }^{4.5}$. It will be interesting to see if reduced intestinal secretory responses can also be demonstrated for CF heterozygotes, as defective regulation of intestinal secretion in CF homozygotes involves not only cyclic AMP, but also calcium ${ }^{h}$ and cyclic GMP ${ }^{7.8}$.

JAN BIJMAN

Hugo De Jonge

Department of Cell Biology and Genetics, Erasmus University, PO Box 1738 , Rotterdam, The Netherlands

JEFFREY WINE

Cystic Fibrosis Research Laboratory,

Bldg 420, Stanford University,

Stanford, California 94305-2130, USA

\footnotetext{
Quinton. P.M. in Fluid and Electrolvte Abnormalities in Exocrine Glands in Custic Fibrosis. (eds Quinton. P. Martinez, R. \& Hopfer, U.) 53 (San Francisco Press, 1982) Hansson. G.C. Nature 333, 711 (1988)

Baxter. P.S. Nature 335, $211(1988)$

Behm. J.K. et ul. Pediat. Res. 22, 271-276(1986)

Sato, K. \& Sato, F. J. lab. clin. Med. 111, 511-518 (1988) Berschneider. R G et al Gastroenterology 92, 1315 (1987) de Jonge. H.R. et al. Pediat. Pulmonol. Suppl. 1, 54 (1987). Bijman. J. et al. in Exocrine Secretion (eds Wong. P. \& Young. J.) 17-19 (Hong Kong University Press, 1988)
} 